\title{
Iatrogenic Blepharoptosis after Cataract Surgery
}

\author{
Hwa Young Oh, Eun Soo Park, \\ Yong Bae Kim \\ Department of Plastic and Reconstructive \\ Surgery, College of Medicine, \\ Soonchunhyang University \\ Bucheon Hospital, Bucheon, Korea
}

No potential conflict of interest relevant to this article was reported.

\begin{abstract}
Eyelid complications after cataract surgery can occur including ectropion, entropion and blepharoptosis. Among them, blepharoptosis has been reported at high rate about $13 \%$. Various causes of ptosis after cataract surgery have been reported. We report the case of three patients treated for blepharoptosis after cataract surgery. All these patients received cataract extraction by a local ophthalmology before they visited our clinics. They presented aponeurotic dehiscence, it was graded as partial and underwent surgical correction by levator aponeurosis advancement. The postoperative course was uneventful. Ptosis was corrected and there was no recurrence during the period of follow-up. All three of them were pleased with the final result. Since post-cataract extraction ptosis is frequent but a preventable complication, understanding the causes of ptosis after surgery is very important. That can help a surgeon prevent its occurrence with careful surgical planning.
\end{abstract}

Keywords Cataract, Cataract extraction, Blepharoptosis

\section{INTRODUCTION}

A cataract is a clouding of the lens inside an eye which leads to a decrease in vision. It is the most common cause of blindness and is conventionally treated with surgery. Cataract surgery is the removal of the natural lens of the eye that has developed an opacification and been replaced with a synthetic lens to restore the transparency of the lens, which has evolved into a highly efficient and predictable surgery.

Eyelid complications including ectropion, entropion and blepharoptosis can occur after cataract surgery $[1,2]$. The incidence of ptosis after cataract surgery has been reported to be as high as $13 \%$ [3]. Factor associated with an increased risk of ptosis after cataract surgery include injection of local anesthetic directly into the eyelid, damage from massage or compression, retrobulbar or peribulbar injection, traction on the eyelid from the eyelid speculum, in-

Received: Aug 2, 2013 Revised: Oct 20, 2013 Accepted: Oct 21, 2013 Correspondence: Eun Soo Park Department of Plastic and Reconstructive Surgery, Soonchunhyang University Bucheon Hospital, 170 Jomaru-ro, Wonmi-gu, Bucheon 420-767, Korea. E-mail: peunsoo@schmc.ac.kr

http://submit.e-aaps.org/

Copyright $\odot 2013$ The Korean Society for Aesthetic Plastic Surgery.

This is an Open Access article distributed under the terms of the Creative Commons Attribution Non-Commercial License (http://creativecommons.org/licenses/by-nc/3.0/) which permits unrestricted non-commercial use, distribution, and reproduction in any medium, provided the original work is properly cited jury resulting from traction on the superior rectus bridle suture, surgical wound, prolonged postoperative patching, perioperative inflammation, and postoperative lid edema [4]. Among them, the use of a bridle suture or rigid lid speculum has been implicated in cataract surgery to cause persistent blepharoptosis with aponeurotic damage $[5,6]$.

Here, we report cases of three patients treated for blepharoptosis after cataract surgery and analyze these cases, as well as review the literature of ptosis mechanism and clinical treatment.

\section{CASE}

Three patients visited out hospital for correction of blepharoptosis after cataract surgery by a local ophthalmology a few months ago. They suffered from increasing visual field obstruction and cosmetic problems. We first performed history-taking and physical examination, including palpebral fissure height, margin to reflex distance (MRD), lid crease height, and levator function. The contralateral eye was also examined and the effect of Herring's law was evaluated by elevating the ptotic eye to uncover any masked ptosis in the contralateral eye in an unilateral case. All three patients had no anatomical deformity, no prior ptosis, no prior ocular surgery except cataract extraction, no history of dry eyes or lacrimal disease, no systemic diseases associated with ptosis, including muscular dystrophy or Horner's syndrome, no history of uveitis or chronic use of topical steroid, no history of botulinum 
toxin injection into periorbital and forehead regions during year before ptosis repair. We planned surgery immediately and performed surgical correction by advancement of the aponeurotic structures to the tarsal plate. First, premarked area of skin was resected with fine scissors, scalpel, or a needle point Bovie. And a small strip of orbicularis was resected. Conservative fat removal or rearrangement was performed depending on patient's condition and underwent plication of the levator aponeurosis to the anterior tarsus. In an intraoperative view, all the patients were presented aponeurotic dehiscence, and it was graded as partial.

\section{Case 1}

A 54-year-old woman visited our outpatient department because of blepharoptosis on the left eyelid. She received cataract extraction by a local ophthalmology 7 month ago. She had no history of

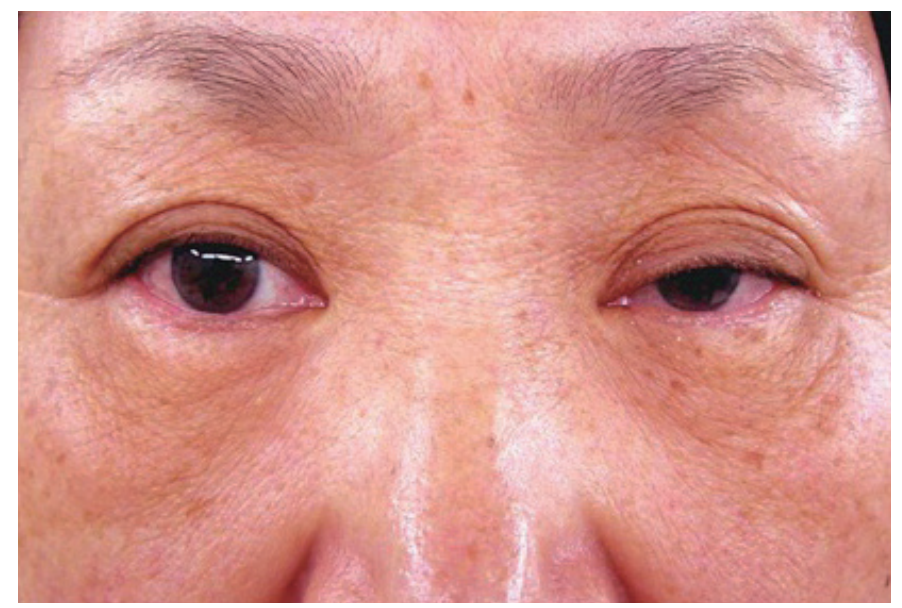

Fig. 1. View of a 54-year-old woman with ptosis of the left upper eyelid 7 months after cataract extraction. Margin to reflex distance is $1 \mathrm{~mm}$. Lid crease height is increased.

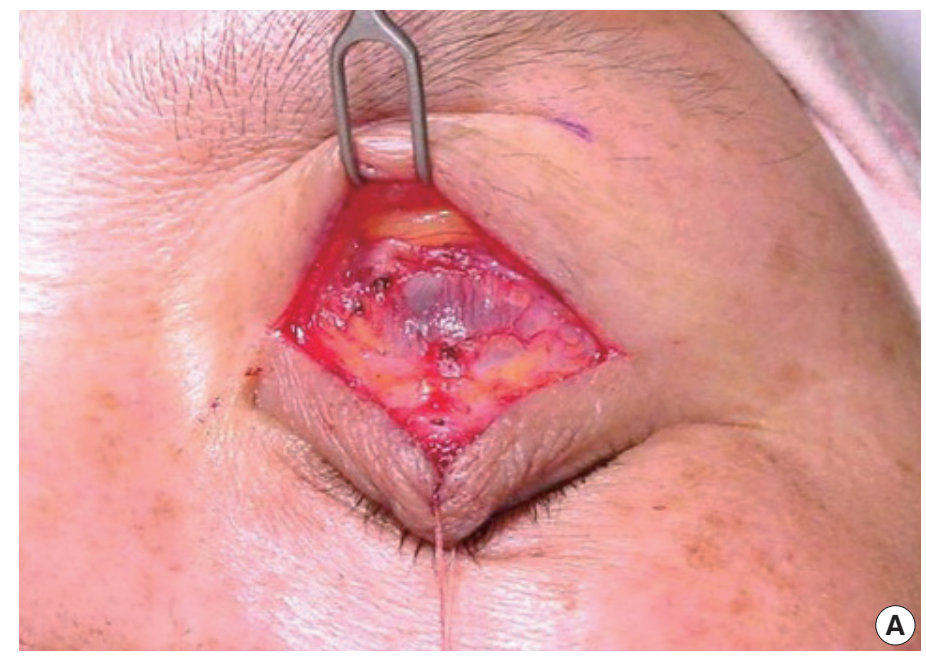

ptosis. She showed that the lid height increase in the left eyelid and a deep superior sulcus (Fig. 1). On the initial evaluation, MRD measured $1 \mathrm{~mm}$ on the left eyelid. Levator function measured 12 $\mathrm{mm}$ on both sides. Contralateral eyelid ptosis was not checked. We performed the levator aponeurosis advancement to the tarsal plate. The postoperative course was uneventful. Ptosis was corrected and no recurrence during the period of follow-up (Fig. 2, 3).

\section{Case 2}

A 72-year-old woman was seen by us for correction of bilateral blepharoptosis after cataract surgery with increasing visual field obstruction. She received cataract extraction by a local ophthalmology 9 month ago. On the initial evaluation, MRD measured -2 $\mathrm{mm}$ on the right eyelid and $0 \mathrm{~mm}$ on the left. She showed severe ptosis on both eyelids and brow ptosis (Fig. 4). We performed the

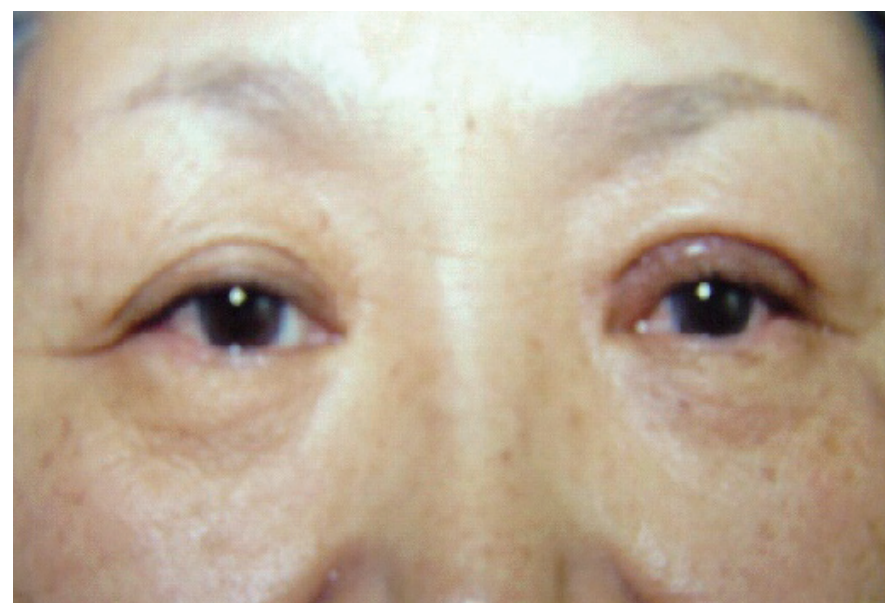

Fig. 3. Postoperative photographic finding after 2 weeks. Ptosis is corrected.

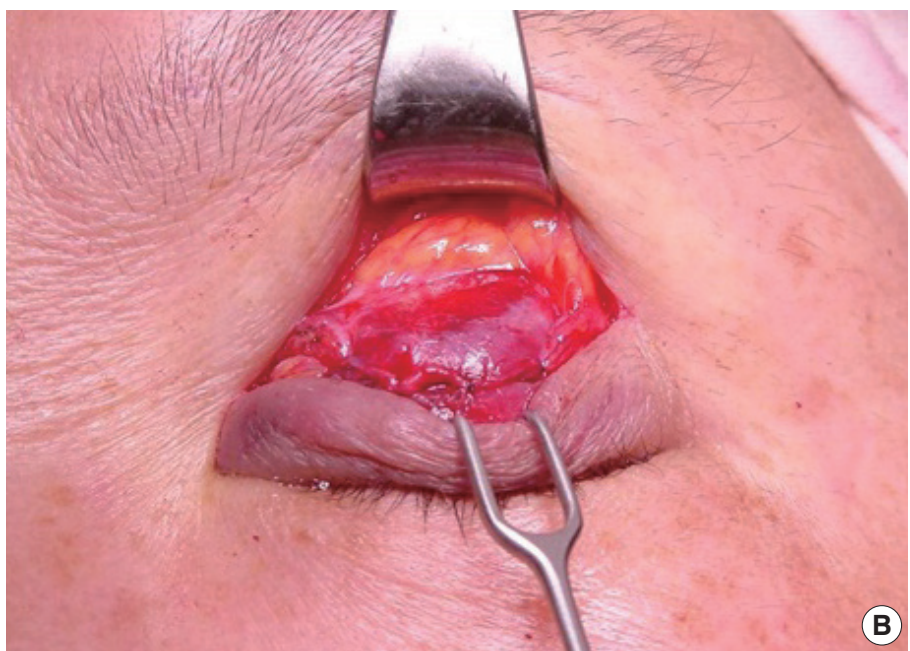

Fig. 2. Intraoperative finding. (A) Dehiscence of the levator aponeurosis from the tarsal plate. (B) Advance levator aponeurosis to the tarsal plate. 


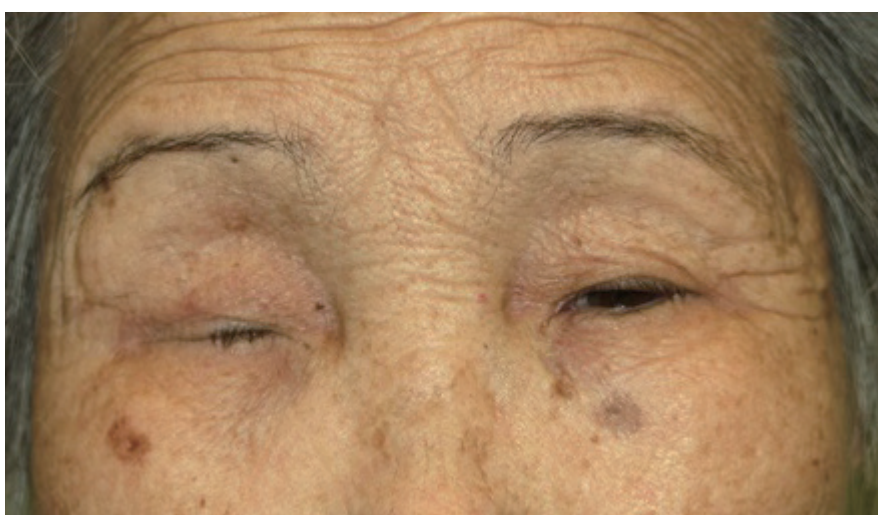

Fig. 4. View of a 72-year-old woman with bilateral blepharoptosis 9 months after cataract extraction and brow ptosis. Margin to reflex distance is $-2 \mathrm{~mm}$ on right eyelid and $0 \mathrm{~mm}$ on left.

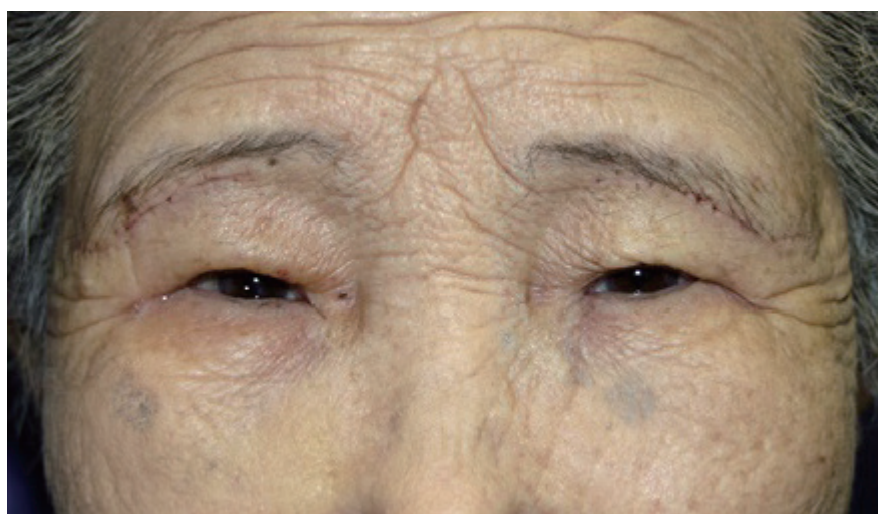

Fig. 5. Postoperative photographic finding after 3 weeks. Ptosis is corrected.

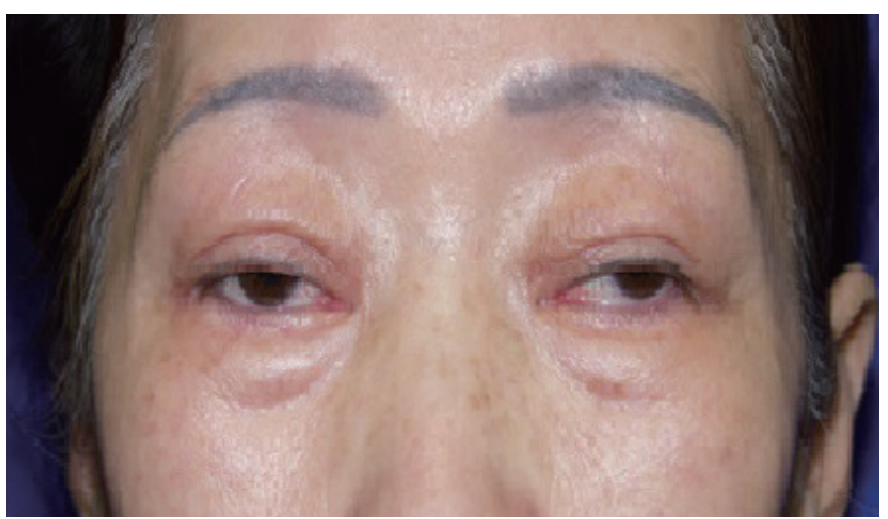

Fig. 6. View of a 52-year-old woman with bilateral blepharoptosis 1 year after cataract extraction.

levator aponeurosis advancement to the tarsal plate and the brow lift for correction of brow ptosis by sub-brow excision. Ptosis was corrected and patient was pleased with final result (Fig. 5).

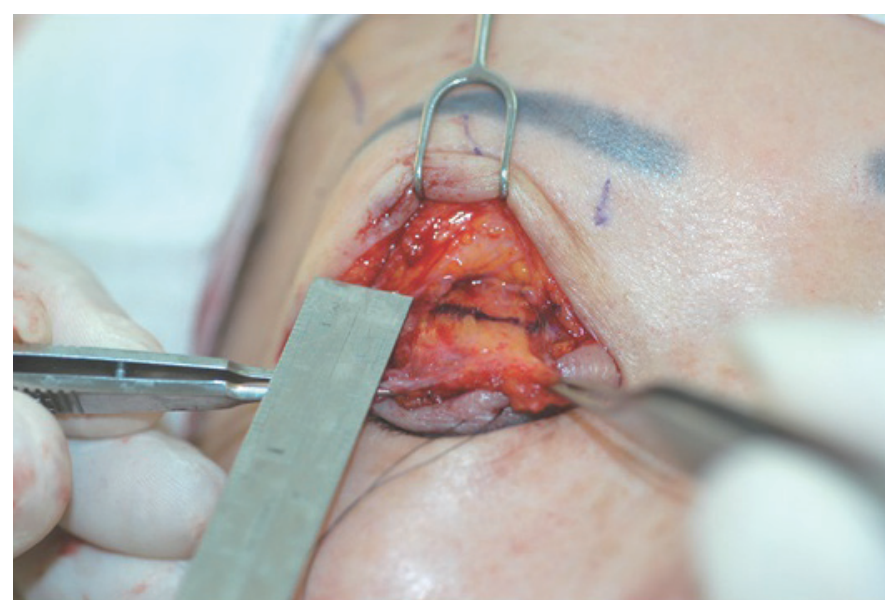

Fig. 7. Intraoperative finding. Advance levator aponeurosis to the tarsal plate.

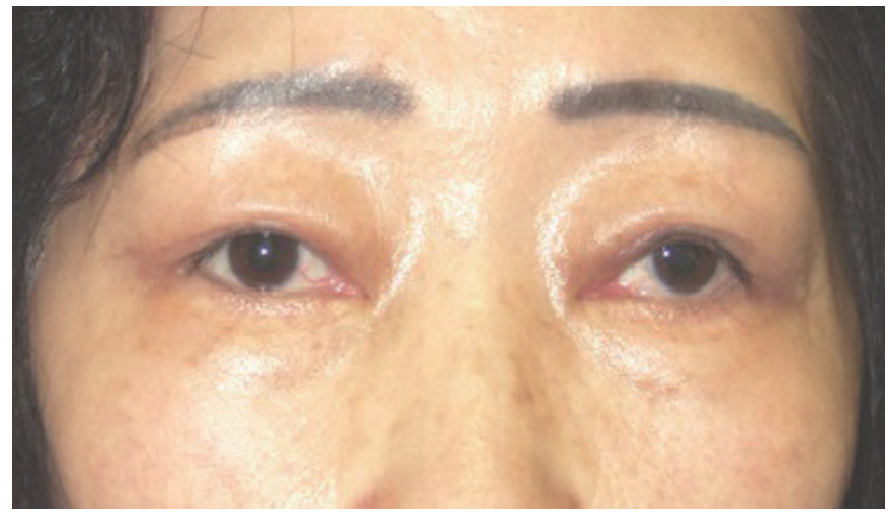

Fig. 8. Postoperative photographic finding after 6 month. Ptosis is corrected.

\section{Case 3}

A 52-year-old woman presented with bilateral blepharoptosis after cataract extraction at 1 year ago. She showed that the lid height increase in both eyelids. On initial evaluation, MRD measured $0 \mathrm{~mm}$ on both eyelids. She had moderate to severe ptosis (Fig. 6). We performed the levator aponeurosis advancement to the tarsal plate. Ptosis was corrected and no visual field defect (Fig. 7, 8) (Table 1).

\section{DISCUSSION}

Common causes of upper lid ptosis are classified into senile, congenital or acquired, and further divided into etiologies including myogenic, aponeurotic, neurogenic, mechanical, or traumatic. Among them, iatrogenic postoperative ptosis is well documented after cataract extraction and other ocular procedures $[7,8]$. Postcataract extraction ptosis may occur from different causes such as grasping the rectus or levator structures, eyelid retraction with 
Table 1. Summary of patients

\begin{tabular}{|c|c|c|c|c|c|}
\hline & & \multicolumn{2}{|c|}{ Preoperation } & \multicolumn{2}{|c|}{ Postoperation } \\
\hline Patient & $\begin{array}{c}\text { Sex/Age } \\
\text { (yr) }\end{array}$ & Right/Left & Right/Left & Right/Left & Right/Left \\
\hline 1 & $54 / F$ & $8 / 5$ & $4 / 1$ & $8 / 8$ & $4 / 4$ \\
\hline 2 & $72 / F$ & $0 / 3$ & $-2 / 0$ & $4 / 4$ & $2 / 2$ \\
\hline
\end{tabular}

bridle sutures or use of an eyelid speculum, injection of local anesthetic solutions into the eyelid structures, eye manipulation, prolonged eye patching, or use of topical steroids [9-11].

Also post-cataract extraction ptosis can be classified into transient ptosis or persistent ptosis by depending on the cause. Transient ptosis can be caused by anesthesia effects, eyelid edema or hematoma, or periocular inflammation. They can occur as a direct damage to the neuromuscular blockade of the levator muscle and the oculomotor nerve fiber. This can be secondary to a retrobulbar or peribulbar block with anesthetic injection to the levator muscle [12]. Also the myotoxic effects of local anesthetics can lead to transient ptosis, which can induce degeneration and subsequent regeneration of muscle fibers of the levator, resulting in weakness that can manifest as ptosis. Postoperative lid edema is probably the most common cause of transient ptosis. It can be secondary to direct infiltration during anesthetic injection, or due to eyelid compression by the speculum against orbital rim. Ocular inflammation may result from suture placement or prolonged wound healing, which can lead to ptosis by promoting prolonged eyelid edema [4]. Transient ptosis are likewise induced by hematoma. Postoperative hematoma can cause mechanical ptosis by mass effect, which pulls down the eyelid. In addition, intraorbital hematoma can cause functional problems of extraocular muscle and malposition of an eyeball. Fibrosis and adhesion from resorbing hematoma can create transient or persistent ptosis [13].

Generally, transient ptosis spontaneously resolves within a few weeks and leaves no complications. Accordingly plastic surgeons should identify the exact cause of ptosis and establish a treatment plans.

Several factors can cause persistent ptosis after cataract surgery, but disinsertion or dehiscence of the levator aponeurosis from tarsal plate is a commonly accepted cause. Generally, levator function should not be affected. Many factors are responsible for the dehiscence of the levator aponeurosis; prolonged lid edema, use of bridle sutures and lid speculum are included.

Kaplan et al. [4] found that placement of a bridle suture with resulting trauma to the superior rectus muscle seemed to be the most important factor in postoperative ptosis. The bridle suture is grasping superior rectus muscle during passage of the needle, which cause inflammation and compression of the vascular supply to the muscle with resulting vascular congestion and stasis, which may disrupt the intermuscular attachments between the levator and superior rectus muscle, then leading to ptosis [6]. Paris and Quickert first hypothesized that prolonged lid edema can cause disinsertion of the levator aponeurosis in older patients who have a vulnerable aponeurosis after ocular surgery [7].

Also the use of lid speculum has been considered as a cause of persistent ptosis. A rigid lid speculum may reduce vascular supply to the levator muscle by compressing the lid against the orbital $\operatorname{rim}[10]$.

Singh et al. [14] recently reported 220 randomized patients who showed significantly higher rate of post-cataract extraction ptosis when a lid speculum was used for retraction along with bridle sutures (44\%) as compared with those in whom only a bridle suture was used without the speculum (23\%).

In postoperative ptosis patients, preoperative history taking and examination are very important. They may be helpful determining of etiology and treatment plan. This includes the preoperative eyelid and ocular condition, history of prior ocular surgery except cataract extraction, history of dry eyes or lacrimal disease, systemic diseases associated with ptosis, including muscular dystrophy or Horner's syndrome, history of botulinum toxin injection into periorbital and forehead regions during year before ptosis repair. The palpebral fissure height, MRD and levator function should be evaluated in both eyelids. Additionally the Herring's test should be performed to identify pseudoretraction of the opposite eyelid [5]. If this occurs, it suggests the presence of bilateral ptosis prior to any ocular surgery.

In this study, one patient is unilateral ptosis and two patients are bilateral. Of the eyelids in this study, all had partial dehiscence that is generally seen in involutional ptosis, and showed normal levator function. We performed post-cataract extraction ptosis repair which is a relatively simple technique requiring identification of the levator aponeurosis and the advancement of levator structures to the tarsal plate. At the same time, brow lift was performed with sub-brow excision in one patient. Ptosis repair was successful in all patients with improving grade.

Post-cataract extraction ptosis is frequent but preventable complication. Understanding the causes of ptosis after surgery is very important that can help a surgeon prevent its occurrence with 
careful surgical planning. Also understanding the causes of ptosis can confer treatment guideline to surgeons which is conservative treatment or surgical correction. Finally, it is crucial to conduct operation with informed consent and patients should be educated about its possibility as a complication following ocular surgery such as cataract surgery, blepharoplasty, orbital bone surgery.

\section{REFERENCES}

1. Hosal BM, Tekeli O, Gürsel E. Eyelid malpositions after cataract surgery. Eur J Ophthalmol 1998;8:12-5.

2. Hurwitz JJ, Smith D, Corin SM. Association of entropion with cataract surgery. Ophthal Plast Reconstr Surg 1990;6:25-7.

3. Alpar JJ. Acquired ptosis following cataract and glaucoma surgery. Glaucoma 1982:466-8.

4. Kaplan LJ, Jaffe NS, Clayman HM. Ptosis and cataract surgery. A multivariant computer analysis of a prospective study. Ophthalmology 1985;92:237-42.

5. Parsa FD, Wolff DR, Parsa NN, et al. Upper eyelid ptosis repair after cataract extraction and the importance of Herring's test. Plast Recontr Surg 2001;108:1527-38.

6. Linberg JV, McDonald MB, Safir A, et al. Ptosis following radial keratotomy. Performed using a rigid eyelid speculum. Ophthal- mology 1986;93:1509-12.

7. Paris GL, Quickert MH. Disinsertion of the aponeurosis of the levator palpebrae superioris muscle after cataract extraction. Am J Ophthalmol 1976;81:337-40.

8. Wolfort FG, Poblete JV. Ptosis after blepharoplasty. Ann Plast Surg 1995;34:264-6.

9. Loeffler M, Solomon LD, Renaud M. Postcataract extraction ptosis: effect of bridle suture. J Cataract Refract Surg 1990;16: 501-4.

10. Ropo A, Ruusuvaara P, Nikki P. Ptosis following periocular or general anesthesia in cataract surgery. Acta Ophthalmol 1992; 70:262-5.

11. Rainin EA, Carlson BM. Postoperative diplopia and ptosis. A clinical hypothesis based on the myotoxicity of local anesthetics. Arch Ophthalmol 1985;103:1337-9.

12. Feibel RM, Custer PL, Gordon MO. Postcataract ptosis. A randomized, doublemasked comparison of peribulbar and retrobulbar anesthesia. Ophthalmology 1993;100:660-5.

13. Bernardino CR, Rubin PA. Ptosis after cataract surgery. Semin Ophthalmol 2002;17:144-8.

14. Singh SK, Sekhar GC, Gupta S. Etiology of ptosis after cataract surgery. J Cataract Refract Surg 1997;23:1409-13. 